Original Article

\title{
Novel method for evaluation of hindfoot alignment in weight-bearing position using laser beam
}

Tadasuke Ohnishi, PT, PO, MS ${ }^{1,2)^{*}}$, Mitsumasa Hida, PT, $\mathrm{MS}^{3)}$, Yukio Nakamura, MD ${ }^{4)}$, Chikamune Wada, $\mathrm{PhD}^{2)}$

1) Department of Rehabilitation, Showa Inan General Hospital: 3230 Akaho, Komagane-shi, Nagano 399-4117, Japan

2) Department of Human Intelligence Systems, Graduate School of Life Science and Systems Engineering, Kyushu Institute of Technology, Japan

3) Osaka Kawasaki Rehabilitation University, Japan

4) Department of Orthopedic Surgery, Shinshu University School of Medicine, Japan

\begin{abstract}
Purpose] It is clinically important to accurately evaluate the alignment of hindfoot alignment from the body surface in weight-bearing position. In general, measurement is carried out using a goniometer, but the measurement with goniometer requires experience, and the reliability in this measurement is also in question. Therefore, it is necessary to establish a clinical measurement method to replace the goniometer. As a solution, we devised a hindfoot alignment measurement method with laser beam (HAML), which measures hindfoot alignment along the body surface using a laser beam. This study aimed to study the correlation between the measurement result along the body surface with laser or goniometer and that of radiography with regard to hindfoot alignment. [Subjects and Methods] Subjects included 23 individuals with knee osteoarthritis. Their hindfoot alignment using radiography and goniometry were measured. The correlation between the measurement result along the body surface with or without laser and radiography was investigated. [Results] The correlation between HAML and radiography was better than that between HAML and goniometry. [Conclusion] The correlation between radiography and HAML for measuring hindfoot alignment was good. This finding implies that HAML can be used as a screening test or epidemiological survey in regional medicine.

Key words: Hindfoot alignment, Weight-bearing position, Laser beam
\end{abstract}

(This article was submitted Nov. 14, 2017, and was accepted Dec. 26, 2017)

\section{INTRODUCTION}

The subtalar joint (STJ), which comprises the hindfoot, performs complex movements to absorb shock and generate propulsive force in a gait cycle ${ }^{1)}$. Studies have reported that the hindfoot adopted to the pathological pattern of the knee joint, such as varus or valgus deformity and patellofemoral pain, which cause mobility abnormality ${ }^{2,3)}$. A specific association between hindfoot malalignment and lower extremity injury has been postulated but not conclusively demonstrated. Many researchers have identified hindfoot malalignment as a risk factor for ankle sprain, stress fracture, and tendonitis ${ }^{4}$. A treatment based on accurate STJ evaluation is important for preventing injuries and re-injuries. In general, hindfoot alignment is evaluated using radiography, with studies reporting that the evaluation has a constant reliability ${ }^{5,6)}$. In particular, the measurement method devised by Saltzman et al. is used to confirm various foot abnormalities ${ }^{7,8)}$. In contrast, some measurement methods along the body surface measure hindfoot alignment. However, a useful and reliable measurement method along the

*Corresponding author. Tadasuke Ohnishi (E-mail: dragoonmouse@gmail.com)

(C2018 The Society of Physical Therapy Science. Published by IPEC Inc.

(c) (1) $\odot$ This is an open-access article distributed under the terms of the Creative Commons Attribution Non-Commercial No Deriva-

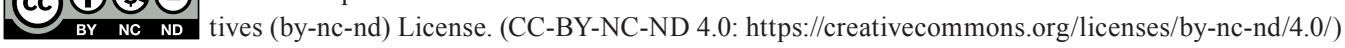


Table 1. Participant characteristics $(n=23)$

\begin{tabular}{lc}
\hline & Mean \pm SD \\
\hline Age (years) & $70.8 \pm 10.3$ \\
Height $(\mathrm{cm})$ & $155.2 \pm 8.4$ \\
Weight $(\mathrm{kg})$ & $55.0 \pm 11.2$ \\
BMI $\left(\mathrm{kg} / \mathrm{m}^{2}\right)$ & $22.7 \pm 3.7$ \\
Gender (male:female) & $3: 20$ \\
\hline
\end{tabular}

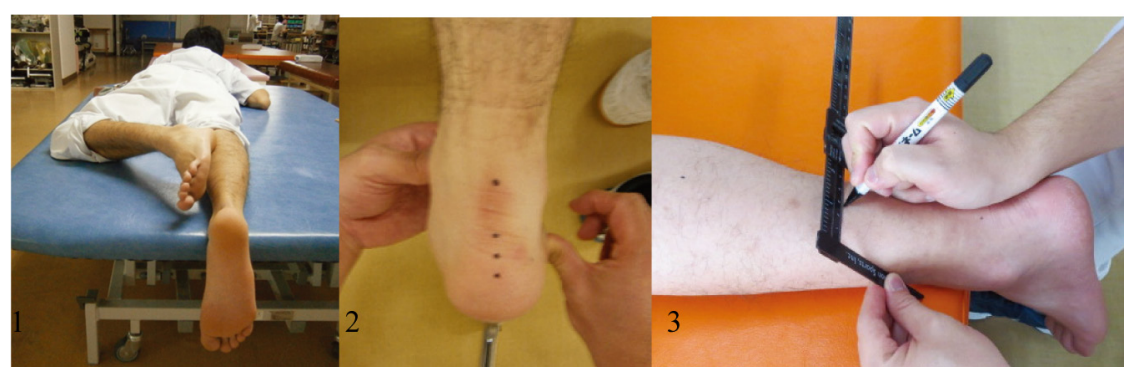

Fig. 1. Posture for marking and marking points.

1) The subject was positioned in prone, with the foot and ankle to be measured hanging over the end of the table; 2) Three points on the bisection line of the calcaneus and one point at Achilles tendon; 3) Two points on the bisection line of the lower leg.

body surface has not been developed because STJ has three joint surfaces with large individual differences ${ }^{1)}$. For example, Elveru et al. reported that they developed a method to measure hindfoot alignment using goniometer, but the result was not reliable $^{9}$. Other study results, such as those of Elveru, lack reliability ${ }^{10,11)}$. However, a study on the correlation between measurement result along the body surface and radiography does not exist. Hence, studying measurement results along the body surface and those from radiography is important because radiography is a standard method for assessing joint malalignment. Therefore, we investigated the correlation between the measurement result along the body surface and radiography in subjects with knee osteoarthritis. Moreover, we devised a novel measurement method using laser beam, namely hindfoot alignment measurement method with laser beam (HAML), as a reliable guide for a perpendicular line. This study aimed to determine the correlation between measurement results along the body surface and radiography in hindfoot alignment. In addition, we focused on the correlation between measurement results from HAML and radiography.

\section{SUBJECTS AND METHODS}

Individuals with knee osteoarthritis were recruited from the medical orthopedic department at our institution. All subjects regularly received medical advice for knee osteoarthritis at the hospital, and they volunteered to participate in the study. Participant characteristics are shown in Table 1.

Exclusion criteria were as follows: (1) past surgery that involved involving the leg, (2) active symptoms of ankle injury or pathology, and (3) inability to symmetrically bear total body weight in the standing position. The measured leg is the right side only. In total, 23 subjects participated in this study. All subjects provided informed consent to participate. The study was approved by the Human Use Review Board at Showa Inan General Hospital of Iowa (No. 2012-10).

The measurement steps along the skin surface are as follows:

1) Marking the body surface.

2) Hindfoot alignment measurement using laser along the body surface.

3) Hindfoot alignment measurement using goniometer along the body surface.

Hindfoot alignment was measured using a laser and goniometer while the patient was standing. Physical therapists with $>5$ years of clinical experience evaluated the subjects. Each subject stood at full-weight bearing on both legs. Before the actual measurement, the patients stood on a scale to attain balance on both legs. Distribution on both legs was defined as within $\pm 3 \mathrm{~kg}$ of the half of the weight. Then, the subject stood with his foot parallel to each other at $10 \mathrm{~cm}$ distance to standardize the posture for radiography.

Marking on the body surface is below:

The marking method was done similar to that of measuring STJ neutral (STJN). For STJN, we used a popular procedure by Elveru et al. ${ }^{12)}$ as reference. We drew some landmarks on the body surface to measure hindfoot alignment (Fig. 1). 
First, we mark three points on the bisection line of the calcaneus and one point at the Achilles tendon. The outline of the calcaneus was palpated to mark them. The three marking points were the most obvious parts of the calcaneus outline above the bottom heel fat pad. We palpated the medial and lateral borders of the calcaneus to mark these three points. We marked dots at each midpoints between medial and lateral border of the calcaneus. These points are measured by using a flexible ruler ${ }^{13,14)}$. These three midpoints on the calcaneus was used for laser measurements and two midpoints at the superior and inferior aspects of the calcaneus was used for goniometric measurements. We then marked the midpoint of the Achilles tendon where the line from the distal end of the medial malleolus to center of the lateral malleolus and the Achilles tendon crossed. Next, we marked the lower leg using a caliper. The caliper was used to identify two midpoints for bisecting the lower third of the leg ${ }^{12,13)}$. The caliper was positioned at the medial and lateral borders of the lower leg approximately $20 \mathrm{~cm}$ and $15 \mathrm{~cm}$ above the calcaneus, and a mark was placed at the midpoint. Midpoint of the Achilles tendon was used for laser measurements and two midpoints for bisecting the lower third of the leg was used for goniometric measurements.

Hindfoot alignment measurement using a laser beam is below:

The laser beam (Cross line laser Quigo; BOSCH Co., Ltd.) was irradiated on the body surface. The laser beam was class 2 in European standard (EN60825-1). The laser beam source was $1 \mathrm{~m}$ away from the heel of the subject. All subjects were informed of the potential risks of the laser. We evaluated the varus-valgus alignment by projecting a vertical laser line from the ground up. The degree of the STJ varus-valgus alignment was divided into seven grades.

Varus or valgus position in STJ was determined according to the laser line. Neutral position implied that every three points in the middle of calcaneus was on the laser line. Strong valgus implied that every three points in the middle was outside of the laser line, moderate valgus was two points outside, and weak valgus was only point outside. These varus positions were also divided into three groups and add neutral position; hence, seven groups were identified (Fig. 2).

Goniometric measurements were completed using a $360^{\circ}$ metal goniometer (arms $30-\mathrm{cm}$ long) marked in $1^{\circ}$ increments. First, we connected two midpoints proximal and distal to the lower third of the leg to make it a bisector. Then, we connected two midpoints in the proximal and distal calcaneus to make it a bisector. An angle was measured when the positions of the lower third of the leg and calcaneus bisecting lines were adjusted using a goniometer.The measurement method showed partial improvements compared with that of Donatelli ${ }^{15)}$ and James ${ }^{16)}$ using the radiography measurement as reference.

The radiographic technique was similar to that originally described by Cobey $\left.{ }^{17}, 18\right)$. The volunteers were placed in a predefined fixed foot position to minimize any influence on the measurements. Both feet were internally rotated to make them parallel. Posture and method used in measurement method for goniometer was similar to that used for laser. The radiographic evaluation method by Saltzman et al. is considered to be the gold standard for assessing hindfoot alignment under loading condition. Hence, radiograph images were evaluated based on the Saltzman hindfoot alignment ${ }^{6,19)}$.

Correlation coefficients between hindfoot alignment measurements with radiograph and HAML were calculated using Spearman's rank correlation coefficient. In addition, the correlation between hindfoot alignment measurements with radiography and goniometry were calculated.The significance level was set at 1\%, and the SPSS software (IBM SPSS Statistics 20.0, IL, USA) was used for statistical processing.

\section{RESULTS}

A significant correlation was observed between HAML and radiography $(\mathrm{r}=0.82 ; \mathrm{p}<0.01)$.

Moreover, a correlation was observed between goniometry and radiography $(r=0.47 ; \mathrm{p}<0.01)$, but the correlation coefficients were higher in HAML than in goniometry. Alignment characteristics of the cohort according to each measurement technique are presented in Table 2. Table 3 shows the correlation between laser method and radiograph, goniometry evaluation.

\section{DISCUSSION}

This study is the first to determine the correlation between measurement results along the body surface and radiography to evaluate hindfoot alignment. And our new hindfoot alignment measurement method developed was good correlation with radiography. To the best of our knowledge, hindfoot alignment evaluation along the body surface was assessed with only interclass correlation coefficients in various evaluation methods. This is big problem because of the inconsistency between joint alignment measurement with radiography and along the body surface ${ }^{18,20,21)}$. This inconsistency can be detrimental to patients, e.g., when he is prescribed a pair of insoles according to his foot deformity. Thus, we used laser beam to evaluate foot alignment to gain a high correlation between radiograph and body surface. The following reasons can be cited because 
Table 2. Alignment characteristics of the cohort according to each measurement procedure

\begin{tabular}{ll}
\hline \multicolumn{1}{c}{ Method } & \multicolumn{1}{c}{ Value } \\
\hline Radiograph, mean $\pm \mathrm{SD},{ }^{\circ}+$ & $1.0 \pm 5.1(-6.54$ to 11.55$)$ \\
Goniometry, mean $\pm \mathrm{SD},{ }^{\circ}+$ & $2.9 \pm 4.4(-10$ to 9$)$ \\
Seven grades of measurement by laser, grade $(\%)^{*}$ & $7(30.4)$ \\
Grade 1 & $0(0)$ \\
Grade 2 & $2(8.7)$ \\
Grade 3 & $2(8.7)$ \\
Grade 4 & $4(17.4)$ \\
Grade 5 & $1(4.3)$ \\
Grade 6 & $7(30.4)$ \\
Grade 7
\end{tabular}

$\dagger$ More than $0^{\circ}$ mean the valgus direction.

*Grade 4 and 5 mean valgus.

Table 3. Correlation between laser method and radiograph, goniometry evaluation

\begin{tabular}{|c|c|c|c|}
\hline \multirow{2}{*}{ Property } & \multicolumn{3}{|c|}{ Coefficient of correlation } \\
\hline & Laser & Radiograph & Goniometry \\
\hline \multicolumn{4}{|l|}{ Laser } \\
\hline Radiograph & $-0.82 * *$ & & \\
\hline Goniometry & -0.37 & $0.47 * *$ & \\
\hline
\end{tabular}

**Significant at the 0.01 level (2-tailed).

we used a laser beam. First, anybody can project a reproducible perpendicular line from the ground when the laser from a correct reference point is projected. Second, we set the reference point of hindfoot alignment at the mid-Achilles tendon. The Achilles tendon attaches to the tuber calcanei, indicating that the mid-Achilles tendon is almost at the center of the talar dome medial to STJ. Thus, at the mid-Achilles tendon, the laser reference point is possibly almost at the center of the talar dome. This reference point is correct and plays an important role in measuring hindfoot alignment.

Moreover, at the mid-Achilles tendon, the laser reference point to measure hindfoot alignment is the line from the medial malleolus distal end to the lateral malleolus center, where the Achilles tendon crosses. Inman reported that the taroclural joint axis almost runs through the distal end of the medial and lateral malleoli ${ }^{22}$, implying that in the reference point in the radiographically hindfoot evaluation method, the center of the talar dome is possibly close to this reference point. In contrast, goniometry has a low correlation. The reference point in goniometry is in the lower leg bisector. The lower leg contains enough soft tissue, and the dimension is asymmetrical even in a healthy individual. This means that the correlation between the lower leg bisector and tibial axis could be low.

A previous study on the interclass correlation coefficient in various evaluation methods revealed that even the result from an experienced examiner was not acceptable ${ }^{23}$. It is easy to imagine that the correlation between measurement using radiograph and that using goniometer is low. Our results are informative for clinicians, and clinicians can obtain a similar result with radiography in hindfoot alignment using the proposed method.

In addition, patients do not need to take a risk for radiation exposure. Our method was an inexpensive and quick measurement method. A treatment based on correct evaluation would be more effective than the current methods used for patients. This study had some limitation. Correlations within an examiner and between examiners were not determined. The measurement procedure was complex, and certain training was required. A manual to correct measurements using the method is required. Furthermore, the subjects were only patients with knee osteoarthritis. The method needs to be used for patients with various pathology patterns and symptoms to establish a further correct method.

\section{Conflict of interest}

The authors have no conflicts of interest to declare.

\section{REFERENCES}


2) Barton CJ, Bonanno D, Levinger P, et al.: Foot and ankle characteristics in patellofemoral pain syndrome: a case control and reliability study. J Orthop Sports Phys Ther, 2010, 40: 286-296. [Medline] [CrossRef]

3) Norton AA, Callaghan JJ, Amendola A, et al.: Correlation of knee and hindfoot deformities in advanced knee OA: compensatory hindfoot alignment and where it occurs. Clin Orthop Relat Res, 2015, 473: 166-174. [Medline] [CrossRef]

4) Larsen E, Angermann P: Association of ankle instability and foot deformity. Acta Orthop Scand, 1990, 61: 136-139. [Medline] [CrossRef]

5) Reilingh ML, Beimers L, Tuijthof GJ, et al.: Measuring hindfoot alignment radiographically: the long axial view is more reliable than the hindfoot alignment view. Skeletal Radiol, 2010, 39: 1103-1108. [Medline] [CrossRef]

6) Saltzman CL, el-Khoury GY: The hindfoot alignment view. Foot Ankle Int, 1995, 16: 572-576. [Medline] [CrossRef]

7) Ward CM, Dolan LA, Bennett DL, et al.: Long-term results of reconstruction for treatment of a flexible cavovarus foot in Charcot-Marie-Tooth disease. J Bone Joint Surg Am, 2008, 90: 2631-2642. [Medline] [CrossRef]

8) Zwipp H, Rammelt S, Endres T, et al.: High union rates and function scores at midterm followup with ankle arthrodesis using a four screw technique. Clin Orthop Relat Res, 2010, 468: 958-968. [Medline] [CrossRef]

9) Elveru RA, Rothstein JM, Lamb RL: Goniometric reliability in a clinical setting. Subtalar and ankle joint measurements. Phys Ther, 1988, 68: 672-677. [Medline] [CrossRef]

10) Picciano AM, Rowlands MS, Worrell T: Reliability of open and closed kinetic chain subtalar joint neutral positions and navicular drop test. J Orthop Sports Phys Ther, 1993, 18: 553-558. [Medline] [CrossRef]

11) Buckley RE, Hunt DV: Reliability of clinical measurement of subtalar joint movement. Foot Ankle Int, 1997, 18: 229-232. [Medline] [CrossRef]

12) Elveru RA, Rothstein JM, Lamb RL, et al.: Methods for taking subtalar joint measurements. A clinical report. Phys Ther, 1988, 68: 678-682. [Medline] [CrossRef]

13) Smith-Oricchio K, Harris BA: Interrater reliability of subtalar neutral, calcaneal inversion and eversion. J Orthop Sports Phys Ther, 1990, 12: 10-15. [Medline] [CrossRef]

14) Vogelbach WD, Combs LC: A biomechanical approach to the management of chronic lower extremity pathologies as they relate to excessive pronation. Athl Train, 1987, 22: 6-16.

15) Donatelli RA: Normal biomechanics of the foot and ankle. J Orthop Sports Phys Ther, 1985, 7: 91-95. [Medline] [CrossRef]

16) James SL, Bates BT, Osternig LR: Injuries to runners. Am J Sports Med, 1978, 6: 40-50. [Medline] [CrossRef]

17) Cobey JC: Posterior roentgenogram of the foot. Clin Orthop Relat Res, 1976, (118): 202-207. [Medline]

18) Cobey JC, Sella E: Standardizing methods of measurement of foot shape by including the effects of subtalar rotation. Foot Ankle, 1981, 2: 30-36. [Medline] [CrossRef]

19) Johnson JE, Lamdan R, Granberry WF, et al.: Hindfoot coronal alignment: a modified radiographic method. Foot Ankle Int, 1999, 20: 818-825. [Medline] [CrossRef]

20) Buck P, Morrey BF, Chao EY: The optimum position of arthrodesis of the ankle. A gait study of the knee and ankle. J Bone Joint Surg Am, $1987,69: 1052-1062$. [Medline] [CrossRef]

21) Saltzman CL, Nawoczenski DA, Talbot KD: Measurement of the medial longitudinal arch. Arch Phys Med Rehabil, 1995, 76: 45-49. [Medline] [CrossRef]

22) Isman RE, Inman VT: Anthropometric studies of the human foot and ankle. Bull Prosthet Res, 1969, 10: 97-129.

23) Haight HJ, Dahm DL, Smith J, et al.: Measuring standing hindfoot alignment: reliability of goniometric and visual measurements. Arch Phys Med Rehabil, 2005, 86: 571-575. [Medline] [CrossRef] 